\title{
Complicações cognitivas pós-operatórias relacionadas à anestesia geral em pacientes idosos
}

\author{
Postoperative cognitive complications related to general anesthesia in elderly patients \\ Complicaciones cognitivas pos operatorias relacionadas con la anestesia general en \\ pacientes ancianos
}

Juliana Visacre Lourenço Santos ${ }^{1 *}$, Amanda Barbiero Grando², Daiane Dias de Moura ${ }^{3}$, Laura Böttcher Lins ${ }^{4}$, Ludmila Albeny Mota Perdigão ${ }^{5}$, Marília Medeiros de Matos ${ }^{6}$, Nathasia Christyelle Rolim de Araújo ${ }^{8}$, Priscilla Evellin de Azevedo Torres ${ }^{7}$, Diego de Matos Silveira 9 .

\section{RESUMO}

Objetivo: Analisar e revisar as principais complicações cognitivas relacionadas ao pós-operatório de pacientes idosos submetidos à anestesia geral. Revisão bibliográfica: $O$ aumento da expectativa de vida se relaciona ao maior número de idosos e ao processo de adoecimento. Dessa forma, configuram um público submetido a procedimentos cirúrgicos com mais frequência. Considerando que a faixa etária acima dos 60 anos apresenta maior taxa de comorbidades e mais chance de reserva cognitiva previamente diminuída, as complicações cognitivas como o delirium pós-operatório e a disfunção cognitiva pósoperatória possuem incidência significativa nesse grupo. Estas complicações podem ser temporárias, permanentes e/ou incapacitantes. Observou-se que cirurgias vasculares, ortopédicas e cardíacas podem ter maior associação com tais distúrbios e que fatores como idade, tempo de permanência hospitalar, comorbidades complexas, dor mal controlada, cirurgia de emergência e perda sanguínea intraoperatória intensa, por exemplo, são elementos que contribuem para a identificação do paciente sob risco. Considerações finais: $O$ manejo do paciente idoso frente a uma indução anestésica deve ser individualizado e monitorado com cautela, na medida que a prevalência de afecções e alterações funcionais relacionadas ao envelhecimento são substanciais.

Palavras-chave: Idoso, Anestesia geral, Complicações cognitivas pós-operatórias.

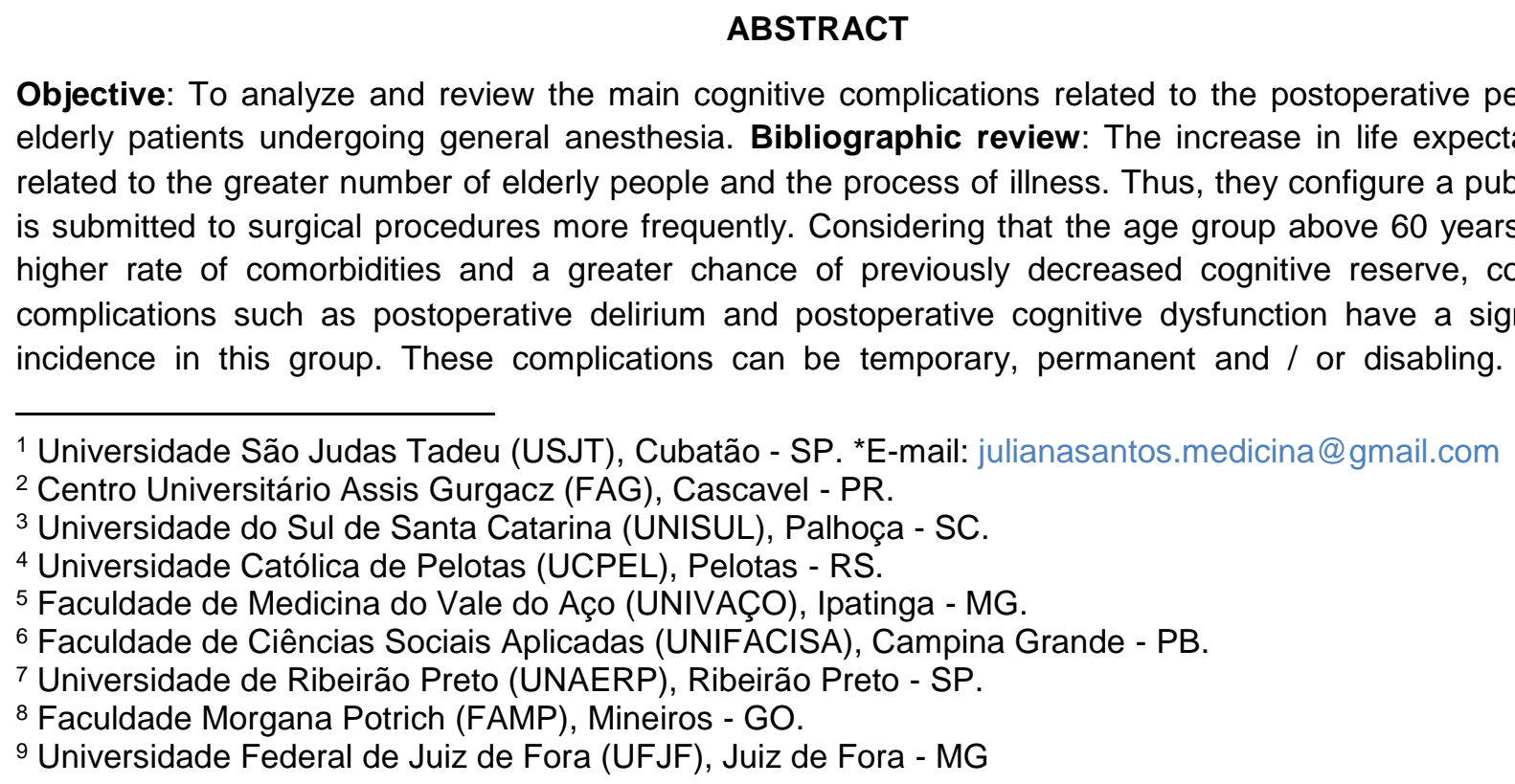


observed that vascular, orthopedic and cardiac surgeries may have a greater association with such disorders and that factors such as age, length of hospital stay, complex comorbidities, poorly controlled pain, emergency surgery and intense intraoperative blood loss, for example, are elements that contribute to the identification of the patient at risk. Final considerations: The management of elderly patients in the face of anesthetic induction should be individualized and carefully monitored, as the prevalence of conditions and functional changes related to aging are substantial.

Keywords: Aged, Anesthesia, General, Postoperative cognitive complications.

\section{RESUMEN}

Objetivo: Analizar y revisar las principales complicaciones cognitivas relacionadas con el postoperatorio de pacientes ancianos sometidos a anestesia general. Revisión bibliográfica: El aumento de la esperanza de vida está relacionado con el mayor número de personas mayores y el proceso de enfermedad. Así configuran un público que se somete a procedimientos quirúrgicos con mayor frecuencia. Considerando que el grupo de edad superior a 60 años tiene una mayor tasa de comorbilidades y una mayor probabilidad de reserva cognitiva previamente disminuida, las complicaciones cognitivas como el delirio postoperatorio y la disfunción cognitiva posoperatoria tienen una incidencia significativa en este grupo. Estas complicaciones pueden ser temporales, permanentes y / o incapacitantes. Se observó que las cirugías vasculares, ortopédicas y cardíacas pueden tener una mayor asociación con dichos trastornos y que factores como la edad, el tiempo de estancia hospitalaria, las comorbilidades complejas, el dolor mal controlado, la cirugía de urgencia y la hemorragia intraoperatoria intensa, por ejemplo, son elementos que contribuyan a la identificación del paciente en riesgo. Consideraciones finales: El manejo de los pacientes ancianos frente a la inducción anestésica debe ser individualizado y monitoreado cuidadosamente, ya que la prevalencia de condiciones y cambios funcionales relacionados con el envejecimiento es sustancial.

Palabras clave: Anciano, Anestesia general, Complicaciones cognitivas postoperatorias.

\section{INTRODUÇÃO}

O estudo da anestesia em seres humanos teve início no século XVIII, quando experimentos com éter surtiram efeitos capazes de bloquear a dor dos pacientes submetidos a procedimentos cirúrgicos. Com o avanço dos estudos, foi possível consolidar a base desta tão importante área da medicina e, a partir de então, houve a difusão destes novos conhecimentos pelo extenso meio científico (AGUILAR MER, 1995).

É evidente a importância das substâncias anestésicas para a realização de procedimentos invasivos, desde aqueles realizados ambulatorialmente até cirurgias de grande porte. Crianças, adolescentes, adultos e idosos são submetidos a procedimentos anestésicos no mundo inteiro, porém, naqueles acima de 60 anos observa-se maior associação às complicações anestésicas pós-operatórias (IRWIN MG, et al., 2019).

Sob a óptica do envelhecimento da população mundial e do número cada vez maior de idosos submetidos a cirurgias, e consequentemente à anestesia, há, de modo diretamente proporcional, elevado risco para o comprometimento cognitivo pós-operatório (SUSANO MJ, et al., 2018). As complicações anestésicas pós-operatórias em idosos podem envolver inúmeros quadros, como hipotensão, alterações de sinais vitais, delírio, agitação, confusão mental e disfunções cognitivas. Os mais vistos na prática médica são o Delírio Pós-Operatório (DPO) e a Disfunção Cognitiva Pós-Operatória (DCPO) (ALDECOA C, et al., 2017).

A DCPO caracteriza-se por prejuízos à memória, à concentração, à compreensão da linguagem e à interação social, que podem ser temporários ou se tornarem permanentes e incapacitantes. Essa complicação está relacionada principalmente à idade do paciente, à presença de Acidente Vascular Encefálico (AVE) prévio, ao histórico de alcoolismo e a cirurgias de grande porte. Sabe-se que a função cognitiva foi, inicialmente, área de estudo de psiquiatras, neurologistas e psicólogos; entretanto, o aumento no número de casos em que há alteração da função intelectual nos pós-operatórios relacionados à 
anestesia geral, enfatiza a importância do estudo e aprofundamento da cognição dos pacientes no período perioperatório (SEKAE T, et al., 2016).

A aplicação de substâncias anestésicas nos casos de anestesia geral pode impactar o paciente idoso de formas que ainda não estão bem esclarecidas. Em alguns casos, além das disfunções cognitivas, pode-se observar relatos de delirium pós-operatório, caracterizado por um quadro confusional mental agudo, possível agitação e comprometimento da cognição. Ainda neste quadro, o paciente pode evoluir com prováveis alucinações e episódios psicóticos. O delirium pós-operatório está mais associado a fatores desencadeantes como estresse, ansiedade e depressão, bem como registra-se maior grau de mortalidade se comparado com os casos de disfunção cognitiva pós-operatória (ALALAWI R e YASMEEN N, 2017).

Considerando, portanto, as mais prováveis complicações anestésicas pós-operatórias em idosos submetidos à anestesia geral, faz-se necessário pontuar a importância da adequada avaliação préoperatória feita pelo médico anestesiologista, com atenção ao histórico médico do paciente, além de um exame físico completo e total monitoramento da evolução do idoso durante o procedimento cirúrgico, visando reduzir as complicações decorrentes dos procedimentos médicos (SUSANO MJ, et al., 2018).

Desse modo, o objetivo deste artigo foi discutir e expor as principais complicações cognitivas no pósoperatório em pacientes idosos submetidos a procedimentos sob anestesia geral, bem como explorar o mecanismo de funcionamento destas desordens.

\section{REVISÃO BIBLIOGRÁFICA}

\section{O estudo da anestesia e seu avanço ao longo do tempo}

O aprendiz de farmácia, Humphry Davy, da cidade de Penzance, na Inglaterra, em 1796, descobriu a propriedade do óxido nitroso em causar ausência de sensibilidade, ao inalá-lo em uma noite que sentia dor de dente. Após inalar o gás, Davy sentiu o total desaparecimento da dor associado a uma sensação de bem-estar. Horace Wells, dentista na cidade de Hartford, quando teve conhecimento do aparente benefício do óxido nitroso, teve a ideia de utilizá-lo em extrações dentárias. Ele primeiro experimentou em si mesmo, solicitou a um seu colega que lhe extraísse um dente, após a inalação do gás. O resultado foi o mesmo obtido pelo aprendiz de farmácia, teve uma sensação de euforia, bem-estar e não sentiu dor (BOBBIO A, 1969; FÜLOP-MILLER R, 1951).

Quando teve a oportunidade de fazer a demonstração desse fato perante professores e estudantes da Faculdade de Medicina de Harvard não alcançou sucesso. Foi posto para fora como charlatão e impostor, pois o voluntário (um estudante presente) gritou de dor. Wells tentou novamente a utilização do óxido nitroso em sua cidade, porém administrou quantidade excessiva de gás e o paciente apresentou uma parada respiratória, a qual quase o levou a óbito. Desmotivado e sem ânimo, abandonou sua profissão de dentista e as experiências (REZENDE JM, 2009).

Em Boston, William Thomas Green Morton, também dentista, orientado pelo seu ex-professor de química, Charles Thomas Jackson, que recomendou usar somente éter retificado e indicou-lhe o local onde poderia obtê-lo, Morton substituiu o óxido nitroso por éter. Os resultados foram muito superiores aos obtidos com o óxido nitroso, superando as expectativas de uso. Em Massachusetts General Hospital no dia 16 de outubro de 1846, Morton demonstrou sua descoberta. Crawford Williamson Long, reivindicou a sua prioridade nessa descoberta, nos países europeus, em meio ao sucesso de Morton, acusando-o de desonestidade. Long, referia que na cidade de Jefferson, no estado da Geórgia, nos Estados Unidos, ele quem tinha feito a primeira intervenção cirúrgica realizada com anestesia geral pelo éter, em 1841 (LEE JA e ATKINSON RS, 1976; REZENDE JM, 2009).

Após a demonstração pública de cirurgia indolor com éter, de 1846. Oliver Wendell Homes nomeou tal ato como "anestesia" (derivada da palavra grega que significa insensibilidade), definida como um estado no qual o organismo não é mais suscetível a estímulos do mundo externo. Na contemporaneidade, é considerado um estado anestesiado, especialmente no contexto cirúrgico, quando, para fins terapêuticos, o indivíduo está em amnésia, com analgesia, imóvel e inconsciente (KELZ MB e MASHOUR GA., 2019). 
Hodiernamente na anestesia geral, ocorre a administração de diferentes medicamentos juntos de maneira balanceada para criar um estado anestésico. Na prática atual é utilizado hipnóticos como Propofol, Éter e Midazolam (esse último, utilizado rotineiramente, antes da indução para diminuição da ansiedade). Os médicos especialistas em anestesiologia criaram essa forma de anestesiar a fim de evitar a dependência exclusiva do éter para a manutenção da anestesia geral. Dessa maneira, administra-se uma quantidade menor de cada medicação, comparada a utilização de uma única droga. Isso aumenta a probabilidade do alcance dos efeitos desejados (inconsciência, amnésia, antinocicepção e imobilidade), e reduz a probabilidade de efeitos colaterais (como por exemplo depressão respiratória, náuseas, vômitos, urinário retenção, constipação e prurido) (BROWN EN, et al., 2018).

Décadas atrás foram estudados diversos componentes e hoje, a prática da anestesia geral se dá por meio de um medicamento hipnótico, como o propofol, para a indução anestésica e a inalação de éter para manter o estado inconsciente do paciente. Apesar de a administração do Midazolam ocorrer frequentemente antes da indução anestésica para tratar a ansiedade, ocorre que seu efeito garante a amnésia, mantendo o paciente no estado de inconsciência (BROWN EN, et al., 2018).

No entanto, mesmo com a criação de diretrizes para prevenção e tratamento de distúrbios cognitivos adversos no pós-operatório devido a existência de comorbidades e mau estado funcional basal do organismo dos pacientes pré-existente, principalmente em idosos, os anestesiologistas ainda vivenciam experiências relacionadas com tal disfunção em seu dia a dia de trabalho (SUSANO MJ, et al., 2018).

\section{Uso de anestesia geral em idosos e suas complicações}

A população geriátrica submetida a cirurgias e à anestesia geral vêm crescendo consideravelmente nos últimos anos. Dados fornecidos pela Organização Mundial da Saúde (OMS), evidenciam que uma parcela considerável das incapacidades decorrentes das doenças no âmbito mundial será tratável por procedimentos cirúrgicos. Esse crescimento significativo relaciona-se com o aumento da expectativa de vida, bem como com a modernização da medicina cirúrgica. Dentre as principais cirurgias realizadas em idosos, têm-se as cirurgias abdominais, ortopédicas, vasculares e plásticas, além de cirurgias no aparelho digestivo, principalmente colecistectomias videolaparoscópicas. No entanto, em intervenções mais agressivas, como no caso de doenças malignas, observa-se certa relutância na recomendação por parte dos médicos, em decorrência da maior morbimortalidade nessa parcela da população (OLIVEIRA DF, et al., 2019).

Dessa forma, ao considerar a grande complexidade que envolve uma cirurgia, é de suma importância levar em consideração as alterações fisiológicas e condições clínicas que cada indivíduo apresenta, principalmente tratando-se de pacientes de maior idade. Todavia, as ferramentas para predição de complicações nesta parcela populacional ainda são insuficientes. Sabe-se que tanto a anestesia, quanto o procedimento cirúrgico em si elevam os riscos de disfunções orgânicas e cognitivas no pós-operatório, como por exemplo, perda de memória e de concentração, confusão mental, alucinações, delírio ou até mesmo distúrbios neurocognitivos discretos, que somente são identificados através de testes neuropsicológicos específicos, como o Mini-Exame do Estado Mental (MEEM), principalmente em pacientes idosos (SUSANO MJ, et al., 2018).

Pode-se destacar, ainda, como exemplos de resultados indesejados: complicações cardíacas ou pulmonares, dor pós-operatória, delírio, agitação de emergência, hipotermia pós-operatória e hiperêmese. Estas complicações também estão relacionadas a maior tempo de permanência hospitalar, declínio funcional, maior morbidade e mortalidade, dependência financeira governamental para auxílio-doença, reinternações e declínio intelectual a longo prazo. Sabe-se que os pacientes na faixa etária geriátrica possuem maior morbidade, mortalidade e número de complicações, quando comparados a adultos, sendo necessárias adaptações corporais e emocionais específicas a esta população (SUSANO MJ, et al., 2018).

Diversas teorias são capazes de explicar essa maior fragilidade em relação aos pacientes idosos, como por exemplo, a própria reserva funcional do indivíduo, que atinge seu pico máximo aos 30 anos e cai gradualmente ano após ano, tendo seu declínio mais acentuado próximo a $8^{\underline{a}}$ década de vida. Essa reserva 
representa uma margem de segurança da capacidade dos órgãos em satisfazer, por exemplo, demandas adicionais de débito cardíaco, síntese de proteínas impostos por trauma ou por doença, ou ainda por um ato mais invasivo como o anestésico-cirúrgico (MENEZES LJ, 2016).

Outras alterações encontradas no organismo dos idosos que poderiam acarretar em situações indesejáveis quanto ao uso de anestésicos que seriam a redução em seu metabolismo basal, alteração na fração livre de fármacos e redução da massa cortical renal de $20-55 \%$, com perda de metade da taxa de filtração glomerular aos 80 anos, o que justifica o fato de que os bloqueadores neuromusculares amplamente utilizados em anestesias gerais, e que apresentam excreção renal, serem utilizados com cautela nessa faixa etária (MENEZES LJ, 2016).

Portanto, é de suma importância frisar as alterações cardiovasculares e pulmonares comuns nessa população previamente, e para as quais os anestesistas devem estar preparados ao lidar com pacientes longevos. A resposta reduzida ao estímulo beta-adrenérgico e às alterações no sistema nervoso autônomo, além de grande labilidade pressórica e eficácia reduzida ao controle barorreflexo, faz dos idosos indivíduos menos capazes de responder à hipovolemia e muito mais susceptíveis a hipotensão ortostática (MENEZES LJ, 2016).

Somado às alterações fisiológicas do idoso como o enrijecimento da parede torácica, enrijecimento da parede arterial, aumento da complacência do parênquima pulmonar, redução na saturação da oxi hemoglobina arterial e da resposta ventilatória à hipóxia, tosse menos eficaz (menor função ciliar) e desordens da deglutição também têm grande importância, já que os episódios de aspiração, principalmente no ato da intubação orotraqueal, são responsabilizados por numerosos casos de pneumonia química e pneumonia pós-operatória (MENEZES LJ, 2016).

\section{Complicações cognitivas consequentes do uso da anestesia}

De maneira geral, a patogênese do comprometimento cognitivo pós-operatório ainda não está muito explicada, porém acredita-se que os agentes anestésicos e métodos anestésicos podem afetar a função nervosa dos pacientes idosos (ZHANG X, et al., 2019). De acordo com Cottrell JE e Hartung J (2020), é suposto que os sedativos e agentes anestésicos agem como neurotoxinas que na maior parte das vezes podem ser reversíveis para pacientes entre 03-60 anos de idade, mas nem sempre isso acontece.

O comprometimento cognitivo pós-operatório é comum em idosos, pois os mesmos apresentam uma reserva cognitiva diminuída pela própria idade. Com isso é sugerido que haja uma trajetória de declínio cognitivo pós-operatório, pois é possível que o delirium pós-operatório leve a alterações microestruturais no cérebro e pode estar associado a subsequentes processos degenerativos, como a demência. Os pacientes que apresentam comprometimento cognitivo pré-operatório têm maior probabilidade de desenvolver 0 delirium pós-operatório e demência posteriormente (STEINMETZ J e RASMUSSEN LS, 2018).

Segundo Aldecoa C, et al. (2017), o Delirium pós-operatório também pode ocorrer em pacientes de qualquer idade, desde crianças a idosos e sua incidência é influenciada por fatores de risco variáveis que se mostram diferentes nas diferentes faixas etárias. Em crianças, as que são mais acometidas estão em idade pré-escolar (5-7 anos) e relacionadas a problemas psicológicos relacionados à idade ou a efeitos inflamatórios no cérebro. Os pacientes idosos são os mais acometidos por apresentarem mais fatores de riscos predisponentes, como redução cognitiva, déficits sensoriais, comorbidades, desnutrição, polifarmácia, estado funcional prejudicado e fragilidade.

A incidência de acometimento do delirium pós-operatório é de $53,3 \%$ em idosos (risco mais elevado); já a disfunção cognitiva pós-operatória possui uma incidência de 20-26\%. Quando ocorre comprometimento cognitivo, ocorre também aumento de complicações pós-operatórias, tempo de internação prolongado (permanência intra hospitalar longa), necessidade de cuidados a um nível mais complexo, persistência da complicação cognitiva pós-operatória e mortalidade pós-cirúrgica (SUSANO MJ et al., 2018).

Sabe-se que a anestesia e a cirurgia podem influenciar de forma negativa no risco de delirium em pacientes idosos, por isso é importante considerar que o anestésico seja escolhido de forma individual, além 
de seu uso ser monitorado, visto que há evidências que mostram que um estado anestésico mais profundo, ou maior tempo de supressão de explosão, podem estar associados, principalmente ao delirium pósoperatório e disfunção cognitiva pós-operatória; e também a importância da realização de avaliação cognitiva pré-operatória de rotina, bem como o uso de monitores para controlar a profundidade da anestesia (SUSANO MJ, et al., 2018).

Além disso, complicações cognitivas pós-operatórias, foi relacionada a idade (maior que 70 anos) a fatores de risco predisponentes a complicações cognitivas pós-operatórias, cirurgia de grande porte, alcoolismo, Acidente Vascular Encefálico (AVE) ou Infarto Agudo do Miocárdio (IAM) prévios como fatores mais preditivos, e sexo masculino, bem como a baixa escolaridade, considerada como fatores de risco de menor importância (SUSANO MJ, et al., 2018).

De acordo com Evered L, et al. (2018), um fato que deve ser levado em consideração é que a incidência relatada de delirium pós-operatório é altamente dependente de como ela é diagnosticada e rastreada, incluindo a forma com que a equipe de avaliação é treinada para a realização da mesma.

O delirium é caracterizado por uma condição neuro inflamatória, que apresenta como características desatenção e instabilidade de consciência (alterando o nível de consciência e atenção a curto prazo, incluindo déficit de memória ou desorientação), podendo acontecer aproximadamente até 30 dias após o procedimento realizado. Geralmente, está associado às cirurgias como cirurgias vasculares, cirurgias ortopédicas e cirurgias cardíacas, sendo diagnosticado clinicamente através do Manual Diagnóstico e Estatístico de Transtornos Mentais V (DSM-V). A etiologia do delirium relacionada à neuroinflamação, refere que quando o tecido neuronal (micróglia e astrócitos) estão expostos a estímulos nocivos e/ou tóxicos, ocorre a liberação de uma cascata de mediadores inflamatórios e citocinas, levando a uma importante lesão neuronal, incluindo danos importantes à barreira hematoencefálica (FITZPATRICK S e OWEN K, 2018).

Os principais fatores predisponentes do delirium são a idade avançada, presença de comorbidades complexas e deficiência cognitiva. Os fatores precipitantes estão relacionados a distúrbios do sono, dores mal controladas, cirurgias de emergência e bem como perda de sangue importante intraoperatória. Identificar o paciente sob risco pode reduzir tanto a gravidade, como a incidência do delirium. $O$ fator idade, o tempo de permanência intra-hospitalar e maior morbidade (quedas, lesões em áreas de pressão e dependência funcional), estão diretamente relacionados ao delirium, e intervenções precoces minimizam suas sequelas (FITZPATRICK S e OWEN K, 2018).

Segundo Cavallari M, et al. (2017), o delirium pode contribuir para o desenvolvimento de anormalidades cerebrais e a sua duração foi associada diretamente a atrofia cerebral global e regional do lobo frontal e hipocampo. Alguns aspectos importantes em relação à anestesia devem ser levados em consideração quando aplicada em idosos, como por exemplo instituir um protocolo específico a pacientes em idades extremas, submetidos a procedimentos cirúrgicos; avaliação pré-operatória adequada e completa de rotina para identificar fragilidades e possíveis intervenções necessárias, além de um acompanhamento direcionado neurocognitivo perioperatório como parte do consentimento informado e esclarecido em pacientes idosos, quando necessário (IRWIN MG, et al., 2019).

\section{Disfunção cognitiva pós-operatória no idoso}

O aumento no número de cirurgias eletivas e cirurgias de emergências em pacientes idosos, juntamente com outros fatores, tem sido associado à DCPO. Nesse contexto, a DCPO consiste em uma deterioração temporária na cognição, associada a um processo como uma cirurgia, sendo quantificada por testes neuropsicólogos. Ademais, estudos apontam como fatores de risco para DCPO: idade avançada, baixo nível intelectual-educacional e suscetibilidade genética. Entretanto, estudos ainda em andamento, buscam a fim de relacionar a exposição a agentes anestésicos com alterações neurodegenerativas persistentes. Desse modo, ainda não existem evidências suficientes que apontem todos os fatores de risco, como também um protocolo específico que indique a conduta correta para prevenção da DCPO (FITZPATRICK S e OWEN K, 2018).

Um estudo, que contrapõe esses dados, foi realizado com um grupo de idosos após a realização de uma cirurgia. Nessa pesquisa, o grupo de idosos, de ambos os sexos, foi acompanhado no ano de 2018 e 
realizou-se uma comparação dos efeitos de técnicas anestésicas utilizadas com o desenvolvimento de doenças cognitivas pós-operatórias. O resultado indicou que a escolha da anestesia não teve a capacidade de desencadear alguma disfunção cognitiva nesses pacientes. Logo, de acordo com esse trabalho, não existem evidências científicas de que uma anestesia é mais benéfica que outra, analisando a sua relação com doenças cognitivas (TZIMAS P, et al., 2018).

\section{CONSIDERAÇÕES FINAIS}

É sabido que o delirium, que é uma condição neuroinflamatória que se caracteriza por desatenção e instabilidade de consciência temporária, é mais prevalente em pacientes idosos submetidos a procedimentos anestésico-cirúrgicos. Este público é mais suscetível a alterações neurodegenerativas persistentes e, por isso, destaca-se a importância de uma boa e completa avaliação cognitiva préoperatória, a escolha correta do tipo de anestesia e o uso de monitores para controlar a profundidade da mesma. Entretanto, não existem evidências científicas que corroboram a prevenção desta complicação, com a finalidade de melhorar o prognóstico e reduzir a morbimortalidade destes pacientes após as abordagens cirúrgicas.

\section{REFERÊNCIAS}

1. AGUILAR MER. Historia y evolución de la anestesia. Cirugia y Cirujanos, 1995; 63(5): 195-198.

2. ALALAWI R, YASMEEN N. Postoperative cognitive dysfunction in elderly: A review comparing the effects desflurane and sevflurane. Journal of Perianesthesia Nursing, 2017; 1-9.

3. ALDECOA C, et al. European Society of Anaesthesiology evidence-based and consensus-based guideline on postoperative delirium. Eur J Anaesthesiol., 2017; 34: 192-214.

4. BOBBIO A. História Sinóptica da Anestesia. São Paulo, Nobel, 1969.

5. BROWN EN, et al. Multimodal general anestesia: theory and Practice. Anesthesia \& Analgesia, 2018.

6. CAVALLARI M, et al. Longitudinal diffusion changes following postoperative delirium in older people without dementia. Neurology, 2017; 89(10): 1020-1017.

7. COTTRELL JE, HARTUNG J. Anesthesia and Cognitive Outcome in Elderly Patients: A Narrative Viewpoint. J Neurosurg Anesthesiol, 2020; 32(1): 9-17.

8. EVERED L, et al. Recommendations for the nomenclature of cognitive change associated with anaesthesia and surgery. Br J Anaesth, 2018; 121: 1005-1012.

9. FITZPATRICK S, OWEN K. Transtornos Cognitivos Pós-Operatórios: Delirium Pós-Operatório e Disfunção Cognitiva Pós-Operatória. Anaesthesia tutorial os the week, 2018; 385: 1-6.

10. FÜLOP-MILLER R. O Triunfo sobre a Dor. História da Anestesia. 2a ed., Rio de Janeiro, José Olympio, 1951.

11. IRWIN MG, et al. Anaesthetic considerations in nonagenarians and centenarians. Curr Opin Anesthesiology, 2019; 32: 776-782.

12. KELZ MB, MASHOUR GA. The biology of general anesthesia from paramecium to primate. Current Biology, 2019; 29: 1199-1210.

13. LEE JÁ, ATKINSON RS. Manual de Anestesiologia. Rio de Janeiro, Livraria Atheneu, 1976.

14. MENEZES LJ. Anestesia em geriatria - revisão de literatura. Dissertação (Residência em Anestesiologia) - Escola de saúde pública do estado do Ceará. Hospital Geral de Fortaleza. Fortaleza, 2016; 28 p.

15. MONK TG, PRICE CC. Postoperative cognitive disorders. National Institute Of Health, 2014; 1-11.

16. TZIMAS $P$, et al. The influence of anesthetic techniques on postoperative cognitive function in elderly patients undergoing hip fracture surgery: General vs spinal anesthesia. Injury, 2018; 49(12): 2221-2226.

17. REZENDE JM. À sombra do plátano: crônicas de história da medicina. São Paulo: Editora Unifesp. Breve história da anestesia geral, 2009; 103-109.

18. REZENDE JM. Scielo Books: editora FAP-Unifesp. 2009; 103-109.

19. SEKAE T, et al. Efeitos da Anestesia Geral na Cognição do Idoso. Arquivos Catarinenses de Medicina, 2016.

20. STEINMETZ J, RASMUSSEN LS. Anesthesia and the risk of dementia in the elderly. La Presse Médicale, 2018; 35(66): 1-7.

21. SUSANO MJ, et al. Distúrbios cognitivos adversos no pós-operatório: uma pesquisa nacional de anestesiologistas portugueses. Revista Brasileira de Anestesiologia, 2018; 68(5): 472-483.

22. ZHANG X, et al. Impacts of General and Spinal Anaesthesia on Short-Term Cognitive Function and Mental Status in Elderly Patients Undergoing Orthopaedic Surgery. Journal of the College of Physicians and Surgeons Pakistan, 2019; 29(2): 101-104. 